\title{
EEG-Based Repetitive Transcranial Magnetic Stimulation for Treatment of Autism Spectrum Disorder
}

\author{
Hung-Shih Lin ${ }^{1,2}$ and Hsu-Wei Fang ${ }^{1 *}$ \\ ${ }^{1}$ Department of Chemical Engineering and Biotechnology, National Taipei University of Technology, Taiwan \\ ${ }^{2}$ Department of Neurosurgery, Mackay Memorial Hospital, Taiwan
}

*Corresponding author: Hsu-Wei Fang, Department of Chemical Engineering and Biotechnology, National Taipei University of Technology, No. 1, Sec. 3, Zhongxiao E. Rd., Daan Dist, Taipei 106, Taiwan

ARTICLE INFO

Received: 凿 March 24, 2021

Published: 幽 March 31, 2021

Citation: Hung-Shih Lin, Hsu-Wei Fang. EEG-Based Repetitive Transcranial Magnetic Stimulation for Treatment of Autism Spectrum Disorder. Biomed J Sci \& Tech Res 34(5)-2021. BJSTR. MS.ID.005623.

Abbreviations: ASD: Autism spectrum disorder; EEG: Electroencephalography; RTMS: Repetitive Transcranial Magnetic Stimulation; QEEG: Quantitative Electroencephalography

\begin{abstract}
Autism spectrum disorder (ASD) is a complex neurodevelopmental disorder with deficits in social communication and interaction, and repetitive behaviors with restricted interests. Some researchers, using electroencephalography (EEG), had identified a reduction of alpha oscillation in the frontal lobe and long-range underconnectivity between the frontal lobe and other regions in alpha oscillation. Both were related to the core symptoms of autism. Repetitive transcranial magnetic stimulation (rTMS) is a non-invasive neuromodulation technique and has been studied for the treatment of individuals with autism. However, there is no consensus on which frequency is suitable. Here, we present a case of autism who received EEGs as evaluations of brain function and individualized transcranial magnetic stimulations that deliver EEG-based alpha-oscillation frequencies. The results were analyzed by quantitative electroencephalography (qEEG) to evaluate the changes of alpha power and connectivity between the frontal lobe and other regions. The EEG results were also compared with clinical evaluations.
\end{abstract}

Keywords: ASD; TMS; qEEG; Coherence

\section{Introduction}

Autism Spectrum Disorder (ASD) is a complex neuro developmental disorder with varying degrees of severity in deficits in social communication and interaction, and repetitive behaviors with restricted interests and activities [1]. In addition to the deficits abovementioned, other associated features include cognitive delay, epilepsy, clumsiness, and psychiatric co-morbidity [2]. Among many measures to identify the neural system abnormalities in individuals with ASD, electroencephalography (EEG) is a convenient and cost-effective method. Research using restingstate EEGs had identified a reduction of frontal alpha power and long-range underconnectivity in alpha oscillations, especially between frontal and other lobes [3]. Since the frontal lobe plays a key role in higher-order cognitive, language, social, and emotional functioning, it is not surprising that deficits in frontal connectivity are related to the core symptoms [4]. Until now, there is no effective pharmacological treatment for core symptoms of ASD and nonpharmacological interventions are costly and time-consuming with modest results. One of the non-invasive neuromodulation techniques that might be effective in the treatment of core symptoms is transcranial magnetic stimulation (TMS) [5]. TMS uses electromagnetic induction to generate a transient and localized electric current in the cortex, causing the firing of neurons. Recently, researchers are using repetitive TMS (rTMS) to study its effect on ASD [6]. Most of the studies used fixed frequency but the frequencies among these studies were different. To date, there is no consensus on which frequency is suitable for most of the patients. Here, we report a case of ASD who received EEGs as evaluations of brain function and individualized rTMS (iTMS) treatments that 
deliver EEG-based alpha-oscillation frequencies based on the calculation of EEGs. The results of EEGs before and after treatments were analyzed by quantitative EEG (qEEG) to identify the changes of the relative power and connectivity which is represented by coherence between tow leads. We also evaluated patient with clinical rating scales.

\section{Case Report}

The patient is a 10-year-old boy with a 7-year history of impairments in social interaction and communication. He also sustained repetitive behaviors and restricted interests. His diagnosis was made according to the Diagnostic and Statistical Manual of Mental Disorders (DSM-V) when he was 3-year-old. In the initial assessment, the Childhood Autism Rating Scale (CARS) score was 47 with severe impairments in listening and visual responses and verbal communication. Also, he scored 40 in Autism Spectrum Quotient (AQ). First, we recorded resting-state eyes-closed EEG by applying two ear electrodes and another 19 electrodes to the scalp in the standard 10-20 International System of Electrode Placement. Data was recorded with Deymed TruScan EEG PT (Deymed Diagnostic s.r.o., Hronov, Czech). After the acquisition of EEG, we analyzed the waves with independent components analysis and discrete Fourier transform and calculated the frequency for TMS treatment. iTMS was administered using a Magstim Rapid2 device (Magstim Corp., Wales, UK) over Fz.
The patient received 25 daily treatments on weekdays in two months with the EEG-based frequency and the power from 60 to $80 \%$ of the motor threshold. In addition to baseline EEG, we followed EEG before the $5^{\text {th }}, 15^{\text {th }}$, and $25^{\text {th }}$ treatment and another EEG on 5 months later. EEG-based frequencies were calculated after every acquisition of EEG then applied to the next treatment. We also evaluated the patient with CARS and AQ when we followed the EEGs. We used Persyst v 13 (Persyst Development Co., Prescott, AZ) to generate qEEG data including relative alpha power of the frontal lobe and alpha-band coherence between frontal and parieto-occipital region. Relative alpha power was calculated by dividing the spectrum power of the alpha-band $(8-13 \mathrm{~Hz})$ by the summation power of all four frequency bands (delta, theta, alpha, and beta bands). Coherence is defined by the synchronization in a fixed frequency band between two leads with the quantification of the extent to which they share a constant oscillating frequency and phase difference [7]. The coherence is a number between 0 to 1 and a higher number indicates more commonality between two channels in a frequency band. We chose 2-minute EEG for each record to calculate relative power at $\mathrm{Fz}$ and coherence between Fp1-P3, Fp1-01, Fp2-P4, and Fp2-02. Clinically, his CARS lowered to 40 and $A Q$ lowered to 35 in the follow-up (Table 1). Initially, the relative power of the frontal lobe was 0.12 and increased to 0.45 at the end of treatment with a reduction to 0.33 at the follow-up (Figure 1). The coherence between bilateral fronto-occipital (F-O) and fronto-parietal (F-P) regions is also increased (Figure 2).

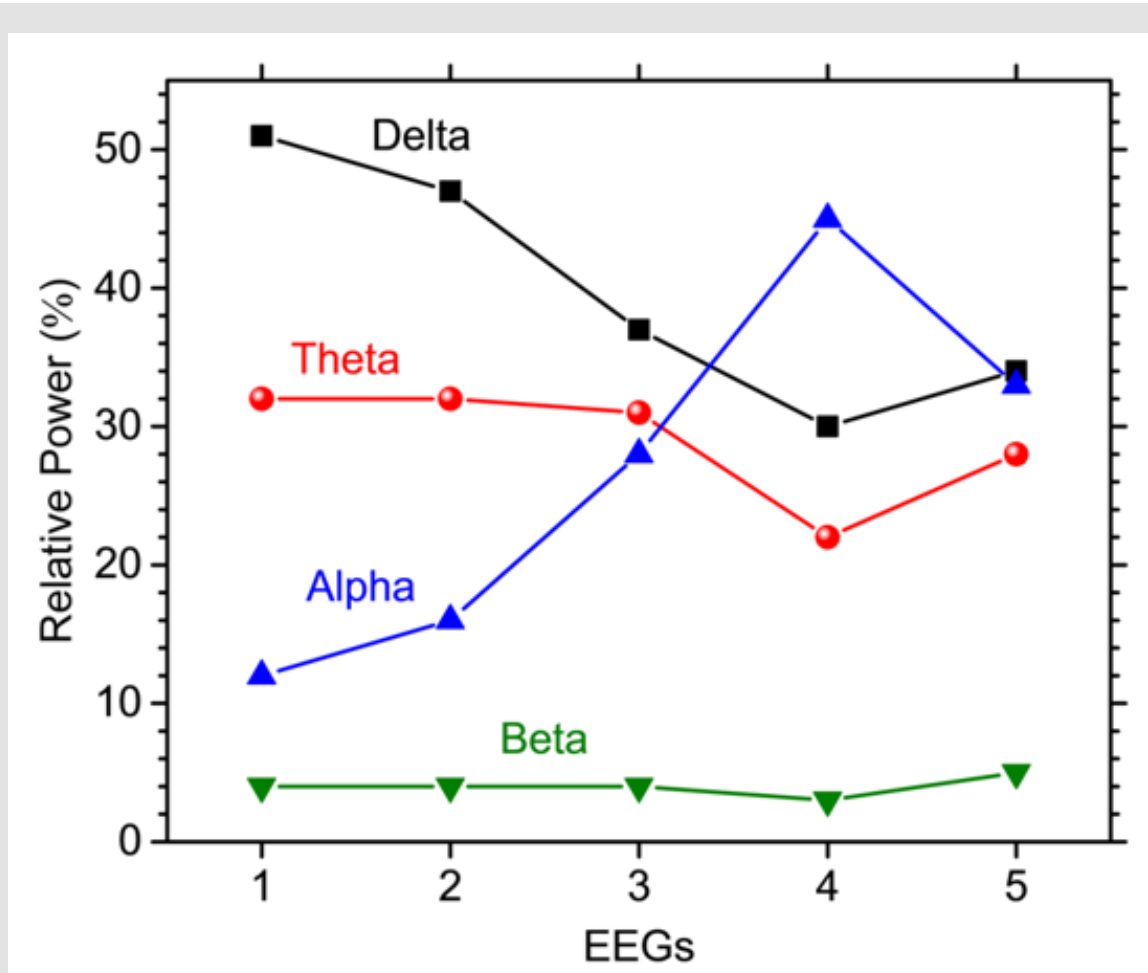

Figure 1: The relative power of different bands of oscillations in the frontal lobe. After treatment, the relative power of alphaband raised obviously while there is a decrement in delta and theta bands. 


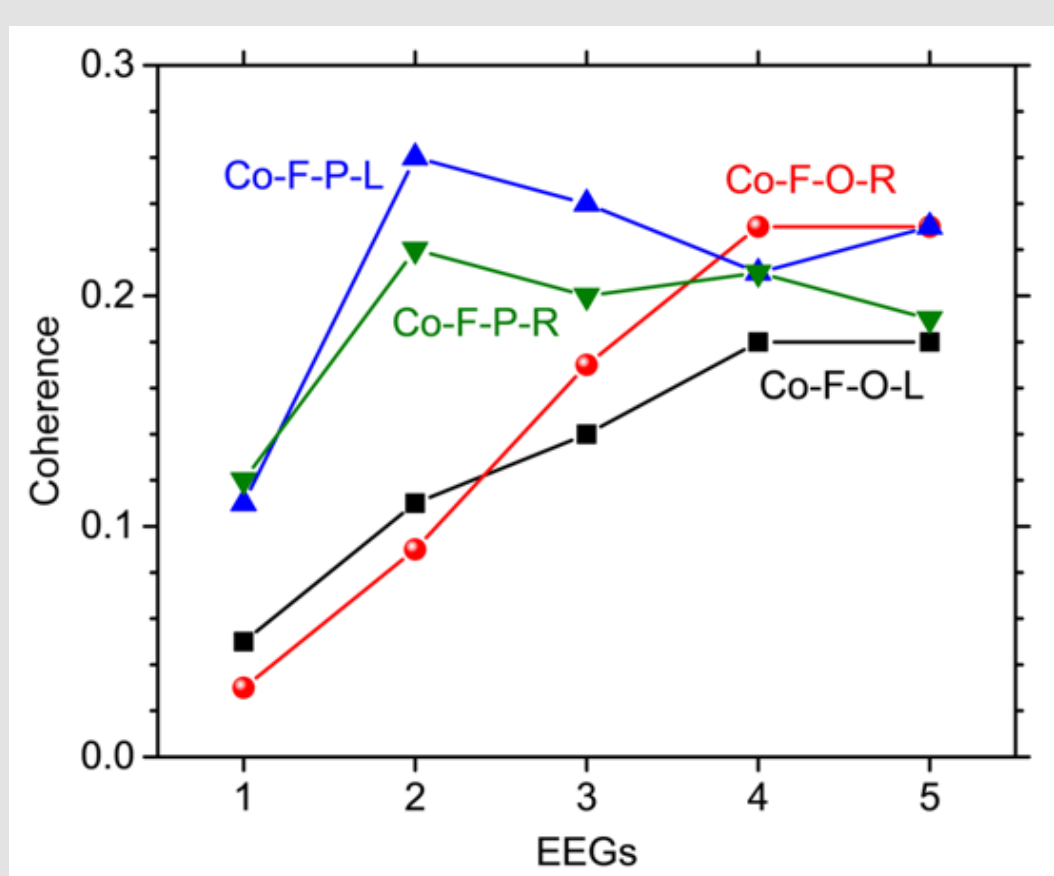

Figure 2: Coherence between the frontal lobe and other regions of the brain. The connectivity between frontal and parietooccipital lobes increased after transcranial magnetic stimulation. Co-F-P-L = coherence between left frontal and parietal lobes; Co-F-P-R = coherence between right frontal and parietal lobes; Co-F-O-L = coherence between left frontal and occipital lobes; $\mathrm{Co}-\mathrm{F}-\mathrm{O}-\mathrm{R}=$ coherence between right frontal and occipital lobes.

Table 1: Different rating scores, the relative power of different oscillations, and coherence between frontal and other lobes before and after the transcranial magnetic stimulation (TMS) treatments.

\begin{tabular}{|c|c|c|c|c|c|}
\hline EEG & $1^{\text {st }}$ & $2^{\text {nd }}$ & $3^{\text {rd }}$ & $4^{\text {th }}$ & $5^{\text {th }}$ \\
\hline (Childhood Autism Rating Scale) CARS & 47 & 47 & 44 & 42 & 40 \\
\hline (Autism Spectrum Quotient) AQ & 40 & 40 & 38 & 37 & 35 \\
\hline Delta relative power $(\%)$ & 51 & 47 & 37 & 30 & 34 \\
\hline Theta relative power (\%) & 32 & 32 & 31 & 22 & 28 \\
\hline Alpha relative power (\%) & 12 & 16 & 28 & 45 & 33 \\
\hline Beta relative power (\%) & 4 & 4 & 4 & 3 & 5 \\
\hline Coherence between left frontal and occipital lobes (Fp1 and 01), Co-F-O-L & 0.05 & 0.11 & 0.14 & 0.18 & 0.18 \\
\hline Coherence between right frontal and occipital lobes (Fp2 and 02), Co-F-O-R & 0.03 & 0.09 & 0.17 & 0.23 & 0.23 \\
\hline Coherence between left frontal and parietal lobes (Fp1 and P3), Co-F-P-L & 0.11 & 0.26 & 0.24 & 0.21 & 0.23 \\
\hline Coherence between right frontal and parietal lobes (Fp2 and P4), Co-F-P-R & 0.12 & 0.22 & 0.2 & 0.21 & 0.19 \\
\hline
\end{tabular}

\section{Discussion}

In the present report, we demonstrate that the changes in qEEGs reflected the results of TMS treatment. For the TMS treatment, the evaluation of the effects depended mainly on clinical evaluation scales and clinical responses without a quantitative tool. qEEG can calculate the oscillation power and connectivity, which are related to the core symptoms in ASD. Therefore, qEEG seems to be an objective measure to evaluate the change in the brain after TMS treatment. In the present case, we found the improvement of the ASD symptoms was accompanied by the increase of alpha power in the frontal lobe. Research had found alpha-band oscillations are the dominant rhythm of the brain and related to attention and basic cognitive process [8]. Many studies in humans using magnetoencephalography and EEG strongly suggested decrement in alpha power facilitated processing in the task-relevant regions and increment of alpha power suppress the task-irrelevant areas, representing the inhibitory property of alpha oscillation. Furthermore, alpha-band oscillation can regulate the change of neuron firing rate which is related to the processing of information by power and phase with higher power with lower firing rate and trough in phase with higher firing rate. Alpha oscillation serves as a regulating system by inhibitory ability to control the firing rate of neurons. So, it is reasonable that increment in alpha power facilitates the control of neuronal firing [9]. 
A previous study had identified long-range underconnectivity between the frontal lobe and other regions of the brain [3], and this deficit was related to core symptoms of ASD since the frontal lobe played a key role in high-order functioning. At any moment, many groups of neurons in our brain are active. It is interesting how they interact with each other and how their communication is flexibly modulated to bring our cognition. Research had found neuronal communication was mechanistically subserved by neuronal coherence. Activated neuronal groups oscillated and underwent rhythmic excitability fluctuations that produced temporal windows for communication. Only coherently oscillating neuronal groups could interact effectively because their communication windows for input and output were open at the same time [10]. So, it is reasonable that increased coherence between the frontal lobe with other regions of the brain means neuronal groups in the frontal lobe communicate more effectively with those in other lobes. Thus, the symptoms due to the deficit of underconnectivity are reduced. Although we demonstrate the relationships between the increase of alpha-band power and coherence to the improvement of ASD symptoms, there is still a drawback here. The alpha-band power of the frontal region was decreased in follow-up, this raises the concern of fade away from treatment. Further follow-up is warranted to confirm the stability of treatment. In this report, we demonstrate the efficacy of TMS and the combination of EEG for the evaluation of results after TMS. Further randomized double-control studies are necessary to verify the increment of frontal alpha power and alpha-band coherence between fronto-parietal and frontooccipital regions are the useful indexes of improvement of clinical symptoms.

\section{Declaration of Patient Consent}

The authors certify that they have obtained all appropriate patient consent forms. In the form the patient(s) has/have given his/her/their consent for his/her/their images and other clinical information to be reported in the journal. The patients understand that their names and initials will not be published, and due efforts will be made to conceal their identity, but anonymity cannot be guaranteed.

\section{References}

1. Volkmar FR, JC Mc Partland (2014) From Kanner to DSM-5: autism as an evolving diagnostic concept. Annu Rev Clin Psychol 10: 193-212.

2. Mouridsen SE, Rich B, Isager T, Nedergaard NJ (2008) Psychiatric disorders in individuals diagnosed with infantile autism as children: a case control study. J Psychiatr Pract 14(1): 5-12.

3. Murias M, Sara W, Jessica G, Geraldine D (2007) Resting state cortical connectivity reflected in EEG coherence in individuals with autism. Biol Psychiatry 62(3): 270-273.

4. Courchesne E, K Pierce (2005) Why the frontal cortex in autism might be talking only to itself: local over-connectivity but long-distance disconnection. Curr Opin Neurobiol 15(2): 225-230.

5. Oberman LM, PG Enticott (2015) Editorial: The safety and efficacy of noninvasive brain stimulation in development and neurodevelopmental disorders. Front Hum Neurosci 9: 544.

6. Barahona Correa JB, Ana V, Ana C, Ricardo L, Albino JO (2018) Repetitive Transcranial Magnetic Stimulation for Treatment of Autism Spectrum Disorder: A Systematic Review and Meta-Analysis. Front Integr Neurosci 12: 27.

7. Mohammad Rezazadeh I, Joel F, Sandra KL, Shafali SJ (2016) Brain connectivity in autism spectrum disorder. Curr Opin Neurol 29(2): 137147.

8. Klimesch W (2012) Alpha-band oscillations, attention, and controlled access to stored information. Trends Cogn Sci 16(12): 606-617.

9. Haegens S, Veronica N, Adrian H, Rogelio L, Ole J, et al (2011) Beta oscillations in the monkey sensorimotor network reflect somatosensory decision making. Proc Natl Acad Sci U S A 108(26): 10708-10713.

10. Fries P (2005) A mechanism for cognitive dynamics: neuronal communication through neuronal coherence. Trends Cogn Sci 9(10): 474-480.
ISSN: 2574-1241

DOI: $10.26717 /$ BJSTR.2021.34.005623

Hsu-Wei Fang. Biomed J Sci \& Tech Res

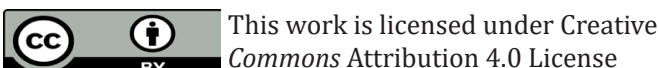

Submission Link: https://biomedres.us/submit-manuscript.php

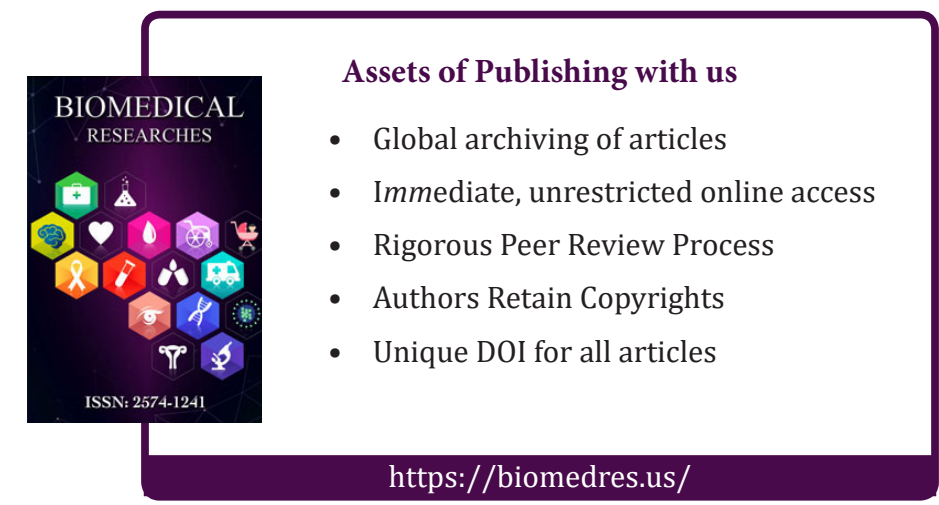

